\title{
Tumor Encasement of the Right Coronary Artery: Role of Anatomic and Functional Imaging in Diagnosis and Therapeutic Management
}

\author{
Yu-Hsiang Juan ${ }^{1,2}$, Yiannis S. Chatzizisis ${ }^{*}, 1,3$, Sachin S. Saboo ${ }^{1}$, Tatiana Rocha ${ }^{1}$ and Michael L. \\ Steigner \\ ${ }^{I}$ Department of Radiology, Brigham and Women's Hospital, Harvard Medical School, Boston, MA, USA \\ ${ }^{2}$ Department of Medical Imaging and Intervention, Chang Gung Memorial Hospital, Linkou and Chang Gung \\ University, Taoyuan, Taiwan \\ ${ }^{3}$ Cardiovascular Division, Brigham and Women's Hospital, Harvard Medical School, Boston, MA, USA
}

\begin{abstract}
We presented two rare cases of mediastinal tumor encasing the right coronary artery (RCA), one with recurrent metastatic thymoma and another with primary poorly differentiated neoplasm. Different degrees and locations of RCA involvement were noted. The treatment approach varied from conservative to surgical. Coronary artery involvement by mediastinal tumors is important to be investigated with imaging as it may guide the surgical planning.
\end{abstract}

Keywords: Computed Tomography, Right Coronary Artery, Tumor, Vascular Encasement.

\section{INTRODUCTION}

Despite the limited current reports of tumor encasement of a coronary artery, coronary encasement can be a lifethreatening condition [1-4] In this work we present two rare cases of right coronary artery (RCA) involvement by a mediastinal tumor.

\section{CASE PRESENTATIONS}

\section{Case 1}

The first case is a 55-year-old woman with mild chest discomfort over several years due to a long-standing history of B3 thymoma. The patient underwent complete tumor resection followed by radiotherapy 12 years ago. She had a tumor recurrence with mediastinal and pericardial invasion and metastasis to both lungs and left subclavian node 7 years ago and she received radical anterior mediastinal resection, as well as resection of the pericardial metastases followed by chemotherapy and radiotherapy. A drug eluting stent was implanted in the RCA due to an incidental injury of the vessel during resection of the pericardial metastasis.

Non-gated multi-detector coronary computed tomography angiography (MDCTA) was performed which visualized the thymoma in the anterior mediastinum, involving the middle and distal RCA (Fig. 1, Panels A-E). Despite the tumor encasement, the RCA remained patent (Fig. 1, Panels A-E). Fluoroscopic coronary angiography was subsequently performed to further confirm the patency

*Address correspondence to this author at the Non-invasive Cardiovascular Imaging Program, Brigham and Women's Hospital, Harvard Medical School, 75 Francis Street, Boston, MA 02115, USA; Tel: +1 857234 2604; Fax: +1 857307 2011; E-mail: ychatzizisis@partners.org of RCA (Fig. 1, Panel F). The patient underwent a debulking surgery to remove as much of the mass as possible while avoiding injuring the RCA. She recovered uneventfully from the surgery and was discharged home with a recommendation of regular follow-up.

\section{Case 2}

The second patient is a 63-year-old woman who was transferred from an outside hospital to our institution for further evaluation and treatment of a recently discovered large anterior mediastinal mass. She complained of several weeks of shortness of breath and vague substernal chest discomfort, worsening with respiration and cough. A routine chest computed tomography (CT) with and without contrast revealed a large anterior mediastinal mass, encasing the entire RCA. The proximal RCA was only visible, whereas the mid and distal RCA were likely occluded by the tumor (Fig. 2, Panel A). The tumor also abutted the proximal ascending thoracic aorta, as well as the right lateral wall of the pulmonary artery. Patient had no signs of myasthenia gravis or B symptoms. Serologic tests revealed elevated lactate dehydrogenase (282 U/L; normal range: 135-225 $\mathrm{U} / \mathrm{L}$ ) and normal values of alpha-fetoprotein and beta-human chorionic gonadotropin, which were not in favor of germ cell tumor clinically. A 18-Flurodeoxyglucose positron emission tomography/computed tomography (18-FDG PET/CT) was subsequently done and showed an intensely avid tumor uptake (Fig. 2, Panel B). An open surgical biopsy was performed which revealed a poorly differentiated neoplasm with necrosis and no evidence of lymphoproliferative disorder. Definitive diagnosis of the tumor type could not be achieved without additional specimen. Total excision of the tumor mass was considered impossible because of its proximity to major vessels and involvement of RCA. The 


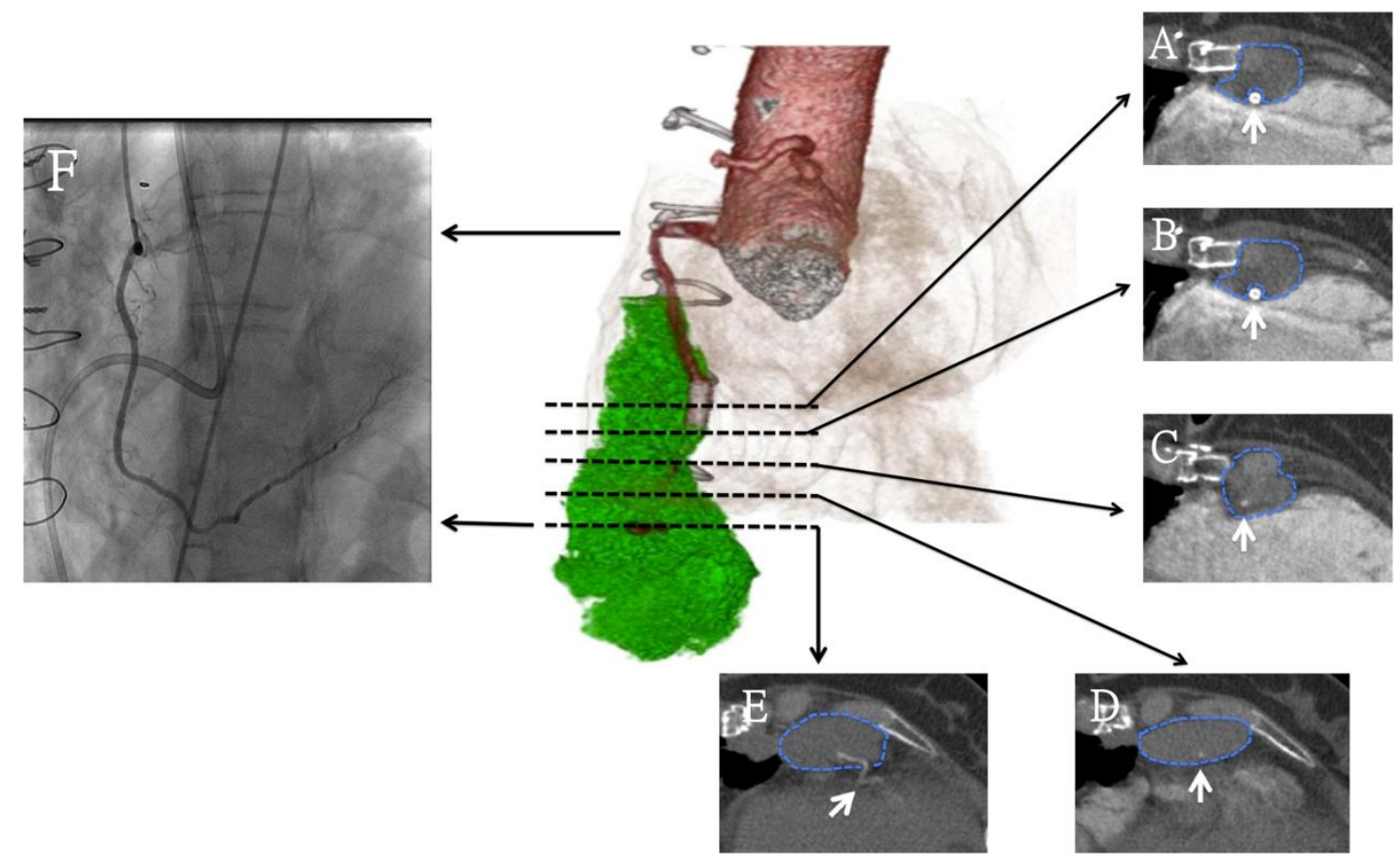

Fig. (1). Chest multi-detector computed tomography and coronary angiography. The volume-rendered 3D image in the center depicts the relationship of the right coronary artery (RCA) with the thymoma. A-E. Corresponding axial images reveal sparing of the proximal stented segment of RCA by the tumor (A) however involvement of distal stented segment in mid RCA (B) to distal RCA segment (C-E). The thymoma is demarcated by the blue dotted line in the axial images. F. Conventional coronary angiogram reveals patent lumen of the RCA.
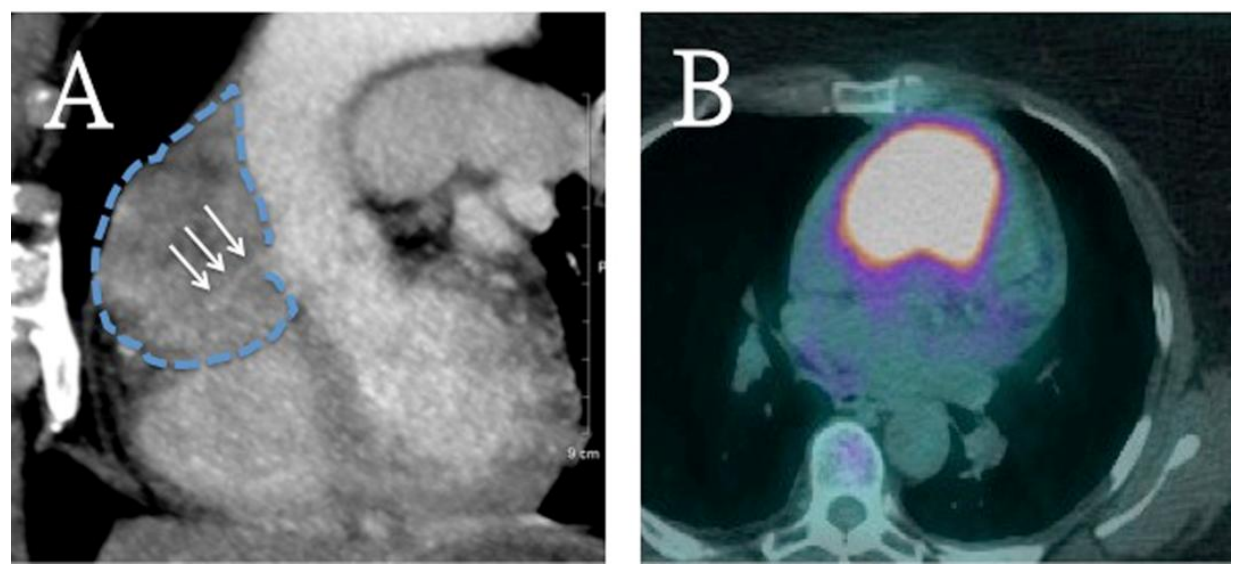

Fig. (2). Chest computed tomography revealing extensive involvement of the RCA by the medistinal tumor with avid tumor uptake on the 18-Flurodeoxyglucose positron emission tomography/computed tomography (PET/CT). A. Sagittal maximum-intensity projection image shows complete encasement of the RCA starting from the orifice, but maintained patency of its proximal segment only (arrow). The tumor is demarcated by the blue dotted line on the sagittal image. B. Axial fused 18 FDG PET/CT reveals significant FDG uptake by the tumor involving the middle mediastinal fat, anterior wall of the ascending aorta, right ventricle outflow tract and pericardium.

patient agreed to receive further clinical follow-up and supportive treatment, but refused to have another biopsy procedure.

\section{DISCUSSION}

In this report we presented two rare cases of a mediastinal tumor encasing the RCA. Different degrees and locations of RCA involvement were noted. The treatment approach varied from conservative to surgical. The first case was a known recurrent thymoma involving the proximal and distal RCA without compromising vessel's patency. The tumor was treated with debulking surgery. Thymoma is a most common anterior mediastnal tumors in adults, but with rare involvement of the heart [5]. It can manifest with myasthenia gravis, compression or encasing of adjacent structures, pleural seedings and paraneoplastic syndromes [6]. The second case was a poorly differentiated mediastinal tumor with encasement of the entire RCA with patency only of its proximal segment. The tumor was treated conservatively due to patient's preference.

The RCA entrapment into a mediastinal tumor has been described as the floating artery sign. This sign has been reported to be specific for diffuse large B cell lymphoma [1, 2], but our case demonstrated that thymoma can also 
demonstrate similar image findings. Diffuse large B cell lymphomas have been reported to frequently encase coronary arteries in the right sided atrioventricular groove [1, 7]. Other types of malignancies, such as angiosarcoma, were shown to cause coronary artery invasion with subsequent pseudoaneurysm or intracoronary thrombus formation [3]. CT is the imaging modality of choice to diagnose, guide therapy and follow-up mediastinal tumors. FDG PET/CT should also be considered, as happened with the second case, especially in case of poorly differentiated tumors.

To the best of our knowledge, there is no evidence-based consensus regarding the treatment of mediastinal tumors encasing coronary arteries. As for the treatment of recurrent thymoma, surgery can improve survival for cases with local recurrence or if complete excision is possible [6]. When complete excision cannot be achieved, like with our first patient, debulking surgery can be considered which, however, has questionable results regarding patient outcomes [6]. Adjuvant radiotherapy and chemotherapy can be also beneficial when radical resection cannot be achieved due to close proximity of the tumor to important vessels or in case of aggressive tumors (B2 or B3) $[6,8]$.

\section{CONCLUSION}

In summary, we demonstrated two cases of RCA involvement by a mediastinal tumor with different degrees and locations of RCA involvements followed by different treatment approaches. RCA entrapment into a mediastinal tumor can manifest as the floating artery sign or with subsequent pseudoaneurysm or intracoronary thrombus formation secondary to intraluminal invasion from more aggressive tumors. Diffuse large B cell lymphomas frequently manifest coronary encasement in the right atrioventricular groove. Coronary artery involvement by mediastinal tumors is important to be investigated with imaging as it may guide the surgical planning. Complete surgical excision can improve survival for cases of recurrent thymomas, and adjuvant radiotherapy and chemotherapy can be also beneficial when radical resection cannot be achieved.

\section{CONFLICT OF INTEREST}

On behalf of all authors, the corresponding author states that there is no conflict of interest.

\section{FUNDING SOURCES}

None

\section{ACKNOWLEDGEMENTS}

None declared

\section{REFERENCES}

[1] Kikuchi Y, Oyama-Manabe N, Manabe O, et al. Imaging characteristics of cardiac dominant diffuse large B-cell lymphoma demonstrated with MDCT and PET/CT. Eur J Nucl Mol Imaging 2013; 40: 1337-44.

[2] Shah RN, Simmons TW, Carr JJ, Entrikin DW. Primary cardiac lymphoma diagnosed by multiphase-gated cardiac CT and CTguided percutaneous trans-sternal biopsy. J Cardiovasc Comput Tomogr 2012; 6: 137-9.

[3] Chaturvedi A, Vummidi D, Shuman WP, Dubinsky TJ, Maki JH Cardiac angiosarcoma: an unusual cause of coronary artery pseudoaneurysm. J Thorac Imaging 2012; 27: W8-9.

[4] Berry MF, Williams M, Welsby I, Lin S. Cardiac angiosarcoma presenting with right coronary artery pseudoaneurysm. J Cardiothorac Vasc Anesth 2010; 24: 633-5.

[5] Thomas A, Shanbhag S, Haglund K, et al. Characterization and management of cardiac involvement of thymic epithelial tumors. J Thorac Oncol 2013; 8: 246-9.

[6] Davenport E, Malthaner RA. The role of surgery in the management of thymoma: a systematic review. Ann Thorac Surg 2008; 86: 673-84.

[7] Minamimoto R, Morooka M, Kubota K, et al. Value of FDGPET/CT using unfractionated heparin for managing primary cardiac lymphoma and several key findings. J Nucl Cardiol 2011; 18: 516-20.

[8] Lucchi M, Mussi A. Surgical treatment of recurrent thymomas. J Thorac Oncol 2010; 5: S348-51. 